\title{
Aeroelastic Characteristics of a Cascade of Mistuned Blades in Subsonic and Supersonic Flows
}

Robert E. Kielb

Lewis Research Center

Cleveland, Ohio

and

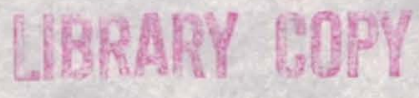

Krishna Rao V. Kaza

The University of Toledo

Toledo, Ohio

\section{AUE 101988}

\section{Prepared for the}

Eighth Biennial Engineering Division Conference sponsored by the American Society of Mechanical Engineers

Hartford, Connecticut, September 20-23, 1981

\section{NASA}




\section{AEROELASTIC CHARACTERISTICS OF A CASCADE OF MISTUNED}

BLADES IN SUBSONIC AND SUPERSONIC FLOWS

by Robert E. Kielb*

National Aeronautics and Space Administration

Lewis Research Center

Cleveland, Ohio 44135

and

Krishna Rao V. Kaza ${ }^{\dagger}$

The University of Toledo

Toledo, Ohio 43606 and

National Aeronautics and Space Administration

Lewis Research Center

Cleveland, Ohio 44135

\section{NOMENCLATURE}

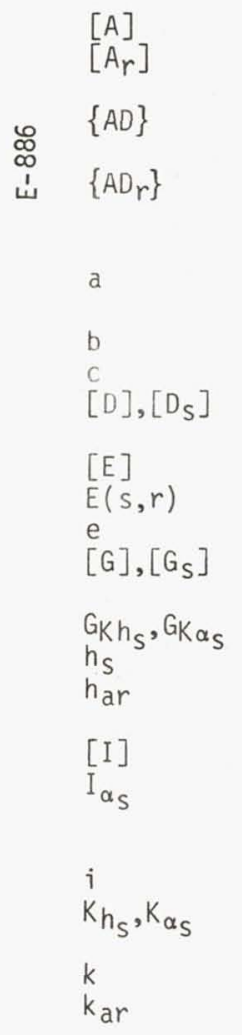

aerodynamic matrix due to motion aerodynamic matrix due to motion in $r$ th mode; $r=0,1,2 \ldots \mathrm{N}-1$ aerodynamic matrix due to wake induced flow

aerodynamic matrix due to wake induced flow in the rth mode, $r=0,1,2, \ldots \mathrm{N}-1$

elastic axis location, nondimensiona]

semichord

chord

matrices defined in Eq. (6); $\mathrm{s}=0,1,2 \ldots \mathrm{N}-1$

matrix defined in Eq. (3)

defined in Eq. (3)

base for natural logarithm

matrices defined in Eq. (6); $\mathrm{s}=0,1,2 \ldots \mathrm{N}-1$

quantities defined in Eq. (6)

bending deflection of sth blade

bending deflection of blade in rth mode of tuned cascade

unit matrix

mass moment of inertia of sth blade about elastic axis per unit span; $\sqrt{-1}\left(=m_{s} r_{\alpha_{s}}^{2} b^{2}\right)$

bending and torsional stiffness

respectively, of sth blade

reduced frequency, $\omega b / V$

acoustic resonance reduced frequency

*Research Engineer, Structural Dynamics Section; Associate member ASME.

†Adjunct Professor, Dept. of Mechanical Engineering, The University of Toledo, Toledo, Ohio.

$k_{F}$
$L_{S}^{M}$
$L_{S}^{M}$
$\ell_{h h r}, \ell_{h \alpha r}$
$\ell_{\alpha h r}, \ell_{\alpha \alpha r}$
$\ell_{W h r}, \ell_{W \alpha r}$
$M_{S}^{M}$
$M_{S}^{W}$
$M_{S}$
$m_{S}$
$[P]$
$r$
$r_{\alpha S}$
$S$
$S_{\alpha S}$
$S$

$\mathrm{t}$
$\mathrm{V}$

$V_{F}$

$\left\{\begin{array}{l}W_{r} \\ X\end{array}\right.$ reduced flutter frequency

lift due to motion of sth blade per unit span, positive up

lift due to wakes of sth blade per unit span, positive up

nondimensional lift coefficients due to bending and torsional motions, respectively, in rth mode

nondimensional moment coefficients due to bending and torsional motions, respectively, in rth mode nondimensional lift and moment coefficients, respectively, due to wake in $r$ th mode

moment about the elastic axis due to motion of sth blade per unit span, positive nose up

moment of sth blade per unit span about the elastic axis due to wake, positive nose up

Mach number

mass per unit span of sth blade

number of blades in cascade

matrix defined in $\mathrm{Eq}$. (6)

integer specifying the mode of tuned rotor; $r=0,1,2 \ldots \mathrm{N}-1$

radius of gyration of sth blade, nondimensionalized with respect to $b$

integer specifying blade, $s=0,1$, $2 \ldots \mathrm{N}-1$; also blade spacing (Fig. 1)

static mass moment of sth blade per unit span about elastic axis, positive when center of gravity is aft of elastic axis

time

freestream velocity relative to the blade

flutter speed

velocity induced by wakes

column matrix, defined in Eq. (3) 


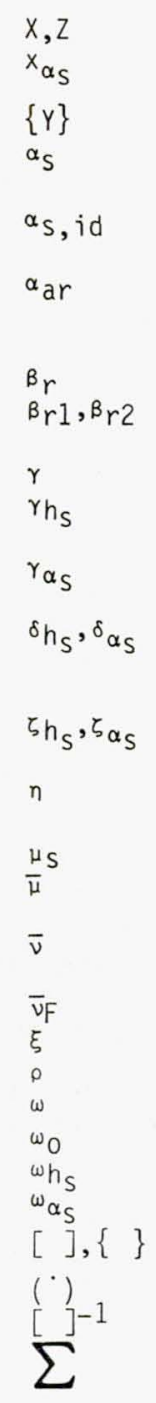

\section{INTRODUCTION}

The prediction of aeroelastic stability and response of bladed-disk assemblies used in aircraft turbofan engines has been receiving considerable attention in the literature. While in most of the research in this area all the blades are assumed identical, a limited amount of work $1-7$ considering either pure bending or torsional motion of the blades has also included small differences between individual blades, known as mistuning. The results in these references have shown a beneficial effect on blade flutter and an adverse effect on forced response. In spite of these findings, the lack of complete understanding of the phenomena has prevented its incorporation in current design analys is systems. Furthermore, the importance of the effects of inherent random mistuning due to manufacturing tolerances and of controlled mistuning upon the aeroelastic characteristics of bladed-disk assemblies have been demonstrated by actual engine experince. $5,8,9$. $A$ research program in turbofan engine aeroelasticity is being conducted in the NASA-Lewis Research Center. As a part of this general program, an effort is in progress to improve the basic understanding of turbofan engine aeroelastic characteristics including mistuning effects. The effects of blade mistuning on coupled bending-torsion flutter and aeroelastic response due to wakes in incompressi- ble flow have been studied in Ref. 10. The purpose of this paper is to continue and to extend the work of Ref. 10 into the subsonic and supersonic flow regimes. To the best of the authors' knowledge, the aeroelastic characteristics of mistuned cascades in subsonic and supersonic flows using a multi-degree of freedom model for the blades have not been studied in the published literature.

Except for the unsteady aerodynamic models, the mathematical model considered is the same as that used in Ref. 10. In the present paper, the unsteady aerodynamic loads are calculated by using Smith's 11 theory in subsonic flow and Adamczyk and Goldstein's 12 theory in supersonic flow with a subsonic leading edge. A brief discussion is presented on three regimes of flutter, which are based on the decaying or propagating nature of an acoustic pressure disturbance in both subsonic and supersonic flows.

\section{THEORY}

\section{A. Structural Model}

As in Ref. 10, the disk is assumed to be rigid and the rotor is modeled as an infinite, twodimensional cascade of airfoils capable of plunging and pitching motions. The geometry of a tuned cascade is shown in Fig. 1. As illustrated in Fig. 2, each airfoil is suspended by bending and torsional springs, $K_{h_{s}}$ and $K_{\alpha s}$ respectively. The blade is assumed to be rigid in the chordwise direction, and, consequently, this motion is neglected. The coupling between bending and torsion due to effects such as pretwist, shrouds, and rotation of the rotor is modeled through the offset distance (b $x_{\alpha_{S}}$ ) between the center of gravity and elastic axis positions. The centrifugal stiffening effects due to rotation are included in the bending and torsional spring constants. The properties of the blades are represented by their respective values at 75 percent span.

In the case of a cascade with identical blades (tuned), it is usually assumed that the motion of the blades is simple harmonic and that they vibrate in each mode of the cascade with a constant amplitude and with a constant phase angle between adjacent blades. The phase angle is restricted to $\mathrm{N}$ discrete values, $B_{r}=2 \pi r / N$, where $r=0,1$, $2 \ldots \mathrm{N}-1$, and thus the total number of allowed interblade phase angle modes of the cascade is also $N$. This restriction is known as Lane's 13 assumption. In the case of a mistuned cascade considered herein, the general motion of a blade can be expressed as a sum of its motions in all possible interblade phase angle modes. Then, the motion of the sth blade is

$$
\left\{\begin{array}{l}
h_{s} / b \\
\alpha_{s}
\end{array}\right\} e^{i \omega t}=\sum_{r=0}^{N-1}\left\{\begin{array}{c}
h_{s} / b \\
\alpha_{a r}
\end{array}\right\} e^{i\left(\omega t+\beta_{r} s\right)}
$$

For a cascade with $\mathrm{N}$ mistuned blades Eq. (1) can be generalized as

$$
\{X\} e^{i \omega t}=[E]\{Y\} e^{i \omega t}
$$

where 


$$
\{x\}=\left\{\begin{array}{l}
h_{0} / b \\
\alpha_{0} \\
\cdot \\
\cdot \\
\cdot \\
h_{N-1} / b \\
\alpha_{N-1}
\end{array}\right\}\{y\}=\left\{\begin{array}{l}
n_{a 0} / b \\
\alpha_{a 0} \\
\cdot \\
\cdot \\
\cdot \\
h_{a(N-1)} / b \\
\alpha_{a(N-1)}
\end{array}\right\}
$$

$[E]=$

$$
\begin{aligned}
& {\left[\begin{array}{llllll}
E(0,0) & 0 & E(0,1) & 0 & \cdots & \\
0 & E(0,0) & 0 & E(0,1) & \cdots & \\
E(1,0) & 0 & \cdots & & & \\
0 & E(1,0) & \cdots & & & \\
\cdot & \cdot & & & & \\
\cdot & \cdot & & E(N-1, N-1) & 0 \\
\cdot & \cdot & & 0 & E(N-1, N-1)
\end{array}\right]} \\
& E(s, r)=e^{2 \pi i s r / N}
\end{aligned}
$$

\section{B. Aerodynamic Model}

As mentioned earlier, the unsteady aerodynamic loads were calculated by using Smith's 11 theory in subsonic flow, and Adamczyk and Goldstein'sl2 theory in supersonic flow with a subsonic leading edge. In these theories, the effect of airfoil thickness, camber, and steady state angle of attack are neglected, and the flow is assumed to be isentropic and irrotational. In the calculation of subsonic aerodynamic loads some numerical convergence problems have been encountered when $\beta_{r}=0$ or $2 \pi$. Since the $B_{r}=0$ or $2 \pi$ mode is of little interest in practice and since a numerical study of this problem is beyond the scope of the present paper, this numerical problem has been resolved by setting $B_{r}=0.001$ whenever its value should be 0 or $2 \pi$. The effect of wakes shed from upstream periodic obstructions are included. The wakes considered are limited to sinusoidal distortions represented by vorticity perturbations so that they are convected downstream at the flow velocity $V$. It should be noted that Ref. 12 does not include the effect of wakes from upstream periodic obstructions. However, the inclusion of this effect in the formulation and the details of the computer program in the supersonic flow have been obtained from Adamczyk.14

\section{Equations of Motion}

The equations of motion for the cascade were developed in Ref. 10. For completeness, those equations are summarized below without the derivation details. The equations of motion for the sth blade are

$$
\left[\begin{array}{ll}
m_{s} & s_{\alpha_{s}} \\
s_{\alpha_{s}} & I_{\alpha_{s}}
\end{array}\right]\left\{\begin{array}{l}
\frac{d^{2}}{d t^{2}}\left(h_{s} e^{i \omega t}\right) \\
\frac{d^{2}}{d t^{2}}\left(\alpha_{s} e^{i \omega t}\right)
\end{array}\right\}+\left[\begin{array}{ll}
\left(1+2 i \zeta_{h_{s}}\right) m_{s} \omega_{h_{s}}^{2} & 0 \\
0 & \left(1+2 i \zeta_{\alpha_{s}}\right) I_{\alpha_{s} \omega_{\alpha_{s}}^{2}}
\end{array}\right]\left\{\begin{array}{l}
h_{s} e^{i \omega t} \\
\alpha_{s} e^{i \omega t}
\end{array}\right\}=
$$

$$
\left\{\begin{array}{l}
-L_{s}^{M}-L_{s}^{w} \\
M_{s}^{M}+M_{s}^{W}
\end{array}\right\}=\left\{\begin{array}{l}
\pi \rho b^{3} \omega^{2} \sum_{r=0}^{N-1}\left[\ell_{h h r} \frac{h_{a r}}{b}+\ell_{h \alpha r^{\alpha} a r}+\ell_{w h r}\right] e^{i\left(\omega t+\beta_{r} s\right)} \\
\pi \rho b^{4} \omega^{2} \sum_{r=0}^{N-1}\left[\ell_{\alpha h r} \frac{h_{a r}}{b}+\ell_{\alpha \alpha r^{\alpha} a r}+\ell_{w \alpha r}\right] e^{i\left(\omega t+\beta_{r} s\right)}
\end{array}\right\}
$$

The aerodynamic coefficients $\ell_{h h r}, \ell_{\text {har }}, \ldots \ell_{\text {war }}$ are functions of the cascade parameters, $k, M, s / c$, $\xi$, a, and $B_{r}$. By nondimensionalizing Eq. (4), extending the resultant equation to all the blades, and using Eq. (2), the equations for all the blades of an arbitrarily mistuned cascade can be written as

$$
\left[[P]-[I]_{\gamma}\right]\{Y\}=-[E]^{-1}[G][E]\{A D\}
$$

where

$$
[P]=\left[[E]^{-1}[D][E]+[E]^{-1}[G][E][A]\right]
$$

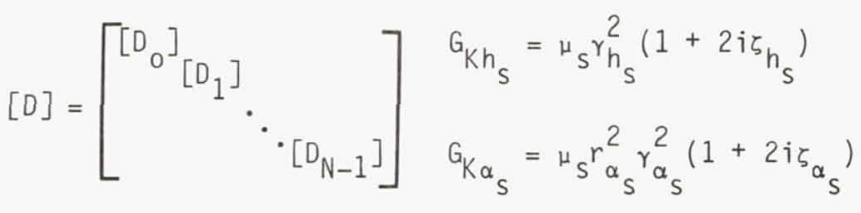

$$
[G]=\left[\begin{array}{lll}
{\left[G_{0}\right]} & & \\
& \left.\ddots G_{1}\right] & \\
& & {\left[G_{N-1}\right]}
\end{array}\right] \begin{aligned}
& \gamma_{h_{s}}=\omega_{h_{s}} / \omega_{0} \\
& \gamma_{\alpha_{S}}=\omega_{\alpha_{s}} / \omega_{0}
\end{aligned}
$$


$[A]=\left[\begin{array}{lll}{\left[A_{0}\right]} & & \\ & {\left[A_{1}\right]} & \\ & \ddots & \\ & & {\left[A_{N-1}\right]}\end{array}\right] \quad \mu_{S}=m_{S} / \pi \rho b^{2}$

$\{A D\}=\left[\begin{array}{llll}\left\{A D_{0}\right. & \left\{A D_{1}\right\} & \cdots & \left\{A D_{N-1}\right.\end{array}\right]^{\top} \quad r_{\alpha_{s}}^{2}=I_{\alpha_{s}} / m_{s} b^{2}$

$\left[D_{s}\right]=\mu_{s}\left[\begin{array}{ll}1 / G_{K h_{s}} & x_{\alpha_{s}} / G_{K h_{s}} \\ x_{\alpha_{s}} / G_{K \alpha_{s}} & r_{\alpha_{s}}^{2} / G_{K} \alpha_{s}\end{array}\right] \begin{aligned} & x_{\alpha_{s}}=S_{\alpha_{s}} / m_{s} b \\ & r=\left(\omega_{o} / \omega\right)^{2}\end{aligned}$

$\left[A_{r}\right]=\left[\begin{array}{ll}\ell_{h h r} & \ell_{h \alpha r} \\ \ell_{\alpha h r} & \ell_{\alpha a r}\end{array}\right]$

$\left\{A D_{r}\right\}=\left[\begin{array}{ll}\ell_{w h r} & \boldsymbol{l}_{\omega \alpha}{ }\end{array}\right]^{\top}$

$\left[G_{s}\right]=\left[\begin{array}{ll}1 / G_{K h_{s}} & 0 \\ 0 & 1 / G_{K \alpha_{s}}\end{array}\right]$

D. Possible Regimes of Flutter

It is obvious that in incompressible flow there are no acoustic (pressure) waves since the governing equation for the perturbation pressure or velocity potential is a Laplace equation. However, in compressible flow acoustic waves are possible since the equation for the perturbation pressure is a 'wave' equation. The possibility of a wave type solution depends on the flow parameters. Different regimes of flutter for subsonic flow were categorized in Ref. 15 based on whether an acoustic disturbance decays or propagates. The marginal condition between these two cases is called the 'acoustic resonance' or 'cutoff'16 condition. For more details see discussions in Refs. 11 and 15. A discussion was presented in Ref. 17 on different regimes of flutter for supersonic flow. To facilitate later discussion of results, a brief description of the possible regimes of flutter in both subsonic and supersonic flow follows.

Subsonic flow. The decaying or propagating nature of an acoustic disturbance can be described by the equation

$$
\left[\left(\frac{B_{r}+2 \pi n}{s / c}\right)^{2}+\frac{4 M^{2}}{M^{2}-1} k\left[k+\left(\frac{B_{r}+2 \pi n}{s / c}\right) \sin \xi\right] \geq 0\right.
$$

which is derived from Eq. (11) of Ref. 11. If the left hand side of Eq. (7) is greater than zero, the disturbances decay exponentially with distance from the cascade and wave type solution for the equation for perturbation pressure is not possible. If flutter occurs when this condition is met, it is called 'subscritical flutter.' If the left hand side of Eq. (7) is less than zero, a wave type solution for the equation for perturbation pressure is possible and at least one pair of acoustic waves can propagate. If flutter occurs when this condition is met, it is called 'supercritical flutter.' If the left hand side of Eq. (7) is zero, a pair of acoustic waves is just on the verge of being able to propa- gate. This marginal condition is called 'acoustic resonance' condition. If flutter occurs very close to acoustic resonance condition, it is called 'acoustic resonance flutter.' The distinction between these regimes of flutter is not always clear cut because they may merge, depending on the system parameters. Also, it should be remarked that this categorization of different regimes of flutter is mainly for descriptive purposes. The value of the integer $n$ in Eq. (7) for the given values of $M, k$, $s / c$, and $\xi$ should be determined such that the roots of the quadratic equation (obtained with equality sign in Eq. (7)), $B_{r 1}$ and $B_{r 2}$, satisfy the condition, $0 \leq \beta_{r} 1,2 \leq 2 \pi$. Alternatively, subcritical and supercritical regimes of flutter can also be categorized based on the values for $B_{r l}$ and $B_{r 2}$. Flutter is called supercritical if the flutter interblade phase angle lies between $\beta_{r} 1$ and $\beta_{r 2}$ and is called subcritical otherwise.

Supersonic flow. The propagating nature of an acoustic disturbance in supersonic flow is also governed by Eq. (7). The acoustic resonance condition obtained from Eq. (7) is the same as the Eq. (48) of Ref. 18. Also, the same resonance condition can be derived from Eq. (1) of Ref. 17. In contrast to the subsonic case, in supersonic flow when the left hand side of Eq. (7) is greater than zero, the acoustic disturbances propagate through the entire flow but remain bounded in the far field. If the flutter occurs when this condition is met, it is called subcritical flutter. If the right side of Eq. (7) is less than zero, the disturbances attenuate in the far field. The flutter, if occurring in this region, is called supercritical.

\section{SOLUTION}

The aeroelastic stability of the cascade is determined by the eigenvalues, $\gamma^{\prime} s$, of the matrix $[P]$ in Eq. (6). The relation between the frequency $\omega$ and $Y$ is

$$
i \omega / \omega_{0}=i / \sqrt{\gamma}=\bar{\mu} \pm i \bar{\nu}
$$

Flutter occurs when $\bar{\mu}>0$. For the given values of the number of blades, and hence the allowable $B r^{\prime} s$, the gap to chord ratio, the stagger angle, the elastic axis position, and the structural parameters, the eigenvalues of the matrix [P] are calculated for a range of values of $k$. Denoting the values of $k$ and $\bar{v}$ at which $\bar{\mu}=0$ as $k F$ and $\bar{v}_{F}$, respectively, the nondimensional flutter speed can be written as

$$
v_{F} / b \omega_{0}=\bar{v}_{F} / k_{F}
$$

The aeroelastic response of the blades induced by wakes is calculated from Eq. (5) and is

$$
\{Y\}=-[[P]-[I] \gamma]^{-1}[E]^{-1}[G][E]\{A D\}
$$

IV. RESULTS AND DISCUSSION

\section{A. Computer Program and Verification}

To calculate the flutter boundaries and the blade aeroelastic response of an arbitrarily mistuned cascade, a digital computer program was written based on the present formulation. In this program, it is possible to consider any type of mistuning such as 
blade to blade variations of the blade uncoupled bending and torsional frequencies, damping ratios, mass ratios, elastic axis and center of gravity positions, and so on. This program is operational on the NASA-Lewis Research Center IBM 370/3033. Both the tuned and mistuned uncoupled bending and uncoupled torsion cases, in addition to the tuned coupled bending-torsion case, can be treated as special cases of this program. This program was checked for the following special cases:

1 . The subsonic aerodynamic coefficients were checked by comparison of the present results to the published results in Refs. 6 and 11. The supersonic aerodynamic coefficients were checked by comparison of the present results to the published results in Refs. 12 and 18.

2. To check the correctness of the program in predicing uncoupled torsional flutter of a tuned cascade in subsonic flow, a 12-bladed rotor described in Ref. 1, was analyzed. A comparison was made of the present results obtained as a special case of the program to those presented in Ref. 15 for the 12-bladed rotor.

3. The correctness of the program in predicting the coupled bending-torsion flutter speed of a tuned cascade in supersonic flow was checked by comparing the present results for a few selected cases to the corresponding ones in Ref. 19.

4. The correctness of the program in calculating coupled bending-torsion flutter and response of a mistuned cascade for $M=0$ was checked by comparing the present results with the corresponding ones in Ref. 10.

In all of these cases good agreement was obtained.

\section{B. Aeroelastic Stability}

Both the aeroelastic stability and response analyses presented in this paper are for NASA Test Rotor 12 (shown in Fig. 3). This special test rotor is similar to a forward stage of an advanced axial flow compressor. The required parameters of this rotor are listed in Table I. It should be mentioned that this rotor was analyzed for incompressible flow in Ref. 10. The blade bending-to-torsion frequency ratio for the tuned rotor is 0.357 , and the elastic axis and c.g. position are at 50 percent chord. As a result, the coupling between bending and torsion is very weak and the flutter mode is dominated by torsional motion. Hence the results for the predominantly bending modes for some cases will not be presented. However, to conduct parametric studies the bending-to-torsion frequency ratio and elastic axis position are varied. For this case the results for the predominantly bending mode will also be presented. Furthermore, no attempt is made to match the flutter Mach number with the assumed Mach number in the parametric study results presented.

As discussed earlier, three different regimes of flutter were found in both subsonic and supersonic flows. It is useful to illustrate them separately. Figures 4 to 6 , show the variation of the real part of the eigenvalue, $\bar{\mu}$ (which is a measure of stability and is defined in Eq. (8)), with the reduced frequency, $k$, at different Mach numbers for a tuned cascade. The flutter mode in these figures is dominated by torsional motion of the blades. In Fig. 4 for $M=0.85$, the regions of subcritical and acoustic resonance flutter and the acoustic resonance reduced frequency, $k_{a r}$, are shown for $B_{r}=83.57^{\circ}$, which is the critical interblade phase angle for subcritical flutter. Also indicated are the acoustic resonance flutter regions for a few other Br's.
No supercritical flutter was found when $M \leq 0.85$. In Fig. 5 for $M=0.9$, the regions of supercritical, acoustic resonance and subcritical flutter, and $\mathrm{k}$ ar are shown for $B_{r}=51.45^{\circ}$ which is the critical interblade phase angle for supercritical flutter. The intervals in which acoustic resonance flutter occurs for a few other values of $B_{r}$ are shown. For a fixed $\beta_{r}$ the subcritical flutter reduced frequency $k_{F}$ is less than the acoustic resonance flutter $\mathrm{KF}_{\mathrm{F}}$, which is less than the supercritical flutter $k_{F}$. The appropriate value of $n$ in determining the regimes of flutter in Figs. 4 and 5 is zero. By comparing Figs. 4 and 5 it can be seen that as $M$ increases toward unity, the regions of acoustic resonance occur at decreasing values of $k$. The frequency separation of these regions also decrease. A similar behavior was observed in Ref. 15 in which only uncoupled torsional motion is considered. In Fig. 6 for $M=1.1$, the regions of supercritical, acoustic resonance, and subcritical flutter are shown. For each $\beta_{r}$ there is a range (a band) in which the supercritical flutter occurs. The width of the band depends on $B_{r}$; and the bands for some $B_{r}$ 's overlap. The appropriate value of $n$ is negative one. No supercritical flutter is found when the Mach number is greater than 1.15 . Thus it is hypothesized that supercritical flutter only occurs when the Mach number is near unity (e.g., $0.85<M<1.15$ ).

A comparison of the system eigenvalues of both tuned and mistuned cascades is useful to understand the effects of mistuning on flutter and response. Figure 7 provides such a comparison for $M=0$. As mentioned earlier, for the parameters considered in this figure the flutter mode is dominated by torsion. As a result, the eigenvalues corresponding to predominantly bending motion are of little interest and are not shown. The type of mistuning considered is the one in which the odd and even numbered blades have different uncoupled torsional frequencies. This is known as alternate blade mistuning. For example, in the case of 1 percent mistuning, the frequency ratio, $\omega_{0} / \omega_{0}$, is 1.005 for all the even blades and is 0.995 for all the odd blades. The reference frequency wo is equal to the arithmetic mean of the uncoupled torsional frequencies of all blades. Because of the symmetry of this type of mistuning the $\beta_{r}$ mode couples with the $\left(\beta_{r} \pm \pi\right)$ mode only. It should be pointed out that the value of the reduced frequency in this figure is chosen such that the cascade is neutrally stable for 2 percent alternate mistuning. Two interesting observations follow from Fig. 7. First, for $M=0$ the 2 percent mistuning significantly affected the system eigenvalues and stabilized the unstable tuned cascade. As the level of mistuning is increased, the spread of the real part of the eigenvalue is decreased. This observation implies that the effective damping of some modes is increased while that of other modes is decreased with an increase in the level of mistuning. This behavior will be recalled in the discussion of forced response which will be addressed later. Second, as mistuning increases the modes separate into high and low frequency groups. The degree of separation increases with the level of mistuning. These findings are in agreement with the similar ones in Ref. 10.

To illustrate the effect of Mach number in the presence of mistuning, the analyses presented in Fig. 7 are repeated in Figs. 8 to 10 at Mach numbers $0.5,1.15$, and 1.4 , respectively. Comparing the ranges of the real parts of the eigenvalues in these figures, several interesting conclusions follow. 
First, this type of mistuning always has a stabilizing effect for the Mach numbers considered. However, the stabilizing effect is stronger in subsonic flow than that in the supersonic flow. Second, in subsonic flow the range of the real parts of the eigenvalues increases with increase in Mach number. But, in the supersonic flow the opposite is true. When $M=1.4$, the effective aerodynamic damping in all the modes is relatively small. Third, the Mach number has a significant effect on the amount of mistuning required to separate the modes into high and low frequency groups. For example, 2 percent mistuning separated the modes in supersonic flow but did not in subsonic flow.

Figure 11 shows how the reduced frequency $\mathrm{kF}$ depends upon Mach number, mistuning, and structural damping. Since the unsteady cascade aerodynamic theories used herein are not valid in the transonic region, no attempt is made to extrapolate the curves in this region. Also, the acoustic resonance flutter regimes are not shown. It is seen that as the Mach number increases from zero, the $\mathrm{kF}_{F}$ of the tuned cascade decreases until supercritical flutter occurs. This decrease in $\mathrm{kF}$ corresponds to efther higher fluld velocities or lower blade stiffness. The effect of increasing $M$ is, therefore, highiy favorable on coupled bending-torsion flutter. In contrast, when $M$ increases from 0.86 to 0.9 , its effect is unfavorable, and the flutter is of supercritical type. When $M$ increases from 1.1 to 1.15 , its effect is again favorable and the flutter is of supercritical type. This increase in $\mathrm{kf}$ corresponds to either lower fluid velocities or higher blade stiffness. Thus, the effect of increasing $M$ on the $k F$ of a tuned cascade depends on the range of M. Also, it is evident from Fig. 11, that the variation of $k F$ with $M$ in an alternately mistuned cascade depends on both the level of mistuning and the range of $M$. The effect of structural damping of a tuned cascade also depends on the range of $M$. Structural damping is highly effective when $M$ is less than 0.6 and less effective when $M$ is greater than 0.6 . In subsonic flow all levels of mistuning considered have much stronger effect on the $\mathrm{kF}$ than does 0.2 percent structural damping. Whereas, in supersonic flow when $M$ is between 1.15 and 1.25 , the 0.2 percent damping is more effective than 2 percent mistuning and is less effective than 5 percent mistuning. When $M$ is between 1.25 and 1.5 , the 0.2 percent damping is more effective than 5 percent mistuning and less effective than 20 percent mistuning. When $M>1.3$, the 0.2 percent damping is more effective than 20 percent mistuning. It should be mentioned that predicted flutter Mach numbers for NASA Test Rotor 12 can be obtained by constructing an 'operating line' in Fig. 11. This operating line was constructed by fixing the speed of sound and the blade uncoupled torsional frequency. The intersection of this line with the neutral stability boundary curve determines the flutter Mach number. Although this line is not shown in Fig. 11, the resulting flutter Mach number is 1.376 for the tuned undamped case. Of course, this value changes if one includes structural damping and blade mistuning. The flutter Mach numbers are 1.396 for 2 percent alternating mistuning, 1.415 for 5 percent mistuning, 1.440 for 20 percent mistuning, and 1.459 for 0.2 percent damped tuned case. All of these flutter Mach numbers are above the design value.

It is apparent from Fig. Il that mistuning has a greater influence in subsonic than in supersonic flow. To determine if this trend shown by Rotor 12 is also similar for a fan stage, a cascade represen- tative of an advanced unshrouded fan stage (aspect ratio 3.34 ) was analyzed. It was found that mistuning had a strong effect on stability at a tip relative Mach number of 1.32 . This effect was similar to that shown by Rotor 12 at low subsonic Mach numbers. Additionally, it appears that the stabilizing effect is sufficient to utilize mistuning as a passive control for unshrouded fan designs in supersonic flow. A detailed description of this analys is will be presented in a future publication.

The effect of the bending-to-torsion frequency ratio, $\omega_{\mathrm{s}} / \omega_{0}$, and elastic axis position on flutter speed, $V_{F}$, is of general interest. To explore these effects, the parameters of the rotor are varied. For example, Fig. 12 shows the dependence of $V_{F}$ of a tuned cascade ( $\omega_{\alpha}=\omega_{0}$ for all s) upon wh $/ w_{0}$ for the elastic axis at 25 percent chord $w i t h M$ as a parameter. When 0 $\leq w_{\mathrm{s}} / \omega_{\mathrm{o}} \leq 0.35$, the Mach number has favorable effect in both subsonic and supersonic flows; when $0.35<\omega_{\mathrm{s}} / w_{0} \leq 1.5, \mathrm{M}$ has unfavorable effect in supersonic flow and favorable effect in subsonic flow; and when $\omega_{h_{S}} / \omega_{0}>1.5, M$ has unfavorable effect in both subsonic and supersonic flows. A very limited study was conducted to determine the effects of alternating structural damping mistuning on aeroelastic stability. The odd numbered blades were left undamped and 0.4 percent structural damping was added to the even numbered blades. For this case with $M=0.5$, the reduced flutter frequency $k_{F}$ is 0.517 . As can be seen from $F i g .11$, this value is very close to the $k_{F}=0.511$ for the case where 0.2 percent damping was added to all the blades equaliy. Also from Fig. 11, the value of $\mathrm{kF}_{\mathrm{F}}$ for the undamped tuned cascade is 0.625 . Comparison of these three values of $k F$ shows that the alternating structural damping does not have any additional benefit over adding damping to all blades equally. Comparison of the eigenvalues, which are not shown due to space limitations, further shows the alternating damping mistuning does not result in the significant mode coupling as was noticed for alternating frequency mistuning.

\section{Aeroelastic Response}

In the present formulation, it is possible to consider an excitation consisting of all harmonics of rotational speed of the rotor which range up to $N-1$. The coefficients, $\ell_{\text {whr }}$ and $\ell_{\text {war }}$, in Eq. (4) represent the forcing functions in the bending and torsional equations, respectively, in the rth mode. To understand the nature of the response, excitation in only one harmonic at a time will be considered. This results in no loss of generality since the principle of superposition holds. If the $r=R$ harmonic is considered, then the column matrices $\left\{A D_{0}\right\},\left\{A D_{1}\right\}, \ldots,\left\{A D_{N-1}\right\}$ are zero except $A D_{R}$ in Eq. (6). According to the traveling wave representation in $\mathrm{Eq}$. (1), this corresponds to N-R symmetrically space obstructions located upstream from the blades and the circumferential wake distribution is perfectly sinusoidal. For practical applications, the forcing frequency is thus equal to $(N-R)$ times the rotational speed.

The aeroelastic response is presented for two values of $R, 11$ (45 obstructions) and 39 (17 obstructions), at a fixed reduced frequency which is chosen such that the cascade is aeroelastically stable in all modes. These values for $R$ were picked because the aerodynamic damping of the tuned system in the $r=11$ mode is relatively low, whereas that in the $r=39$ mode is relatively high. The forcing frequency range investigated is limited to a 
small range around the uncoupled torsional frequency. If the blades are tuned, the response will be entirely in the $r=R$ mode, and all the blades have equal amplitudes. The amplitude of response is a function of $\omega^{\prime} / \omega_{0}$. If the blades are now arbitrarily mistuned, there will be response in all the modes (enumerated by $r$ ) and the response of the sth blade can be obtained from Eq. (1). Figure 13(a) for $R=11$ and $F i g .13(b)$ for $R=39$ show the variation of $\alpha_{S} / \alpha_{S}$, id for both tuned and the two percent alternating blade mistuned cascades in subsonic flow. The quantity $\alpha_{s}$, id is the amplitude of the torsional motion of each blade at resonance in the tuned rotor and it depends upon $R$. Figures $14(a)$ for $R=11$ and $14(b)$ for $R=39$ are repetitions of Figs. 13(a) and (b), respectively, in supersonic flow. The bending amplitudes are not shown because they are very small in the range of the excitation frequency shown herein. For alternating mistuning, only the $\beta_{r}$ and $\left(\beta_{r} \pm \pi\right)$ modes are coupled. Therefore, in all three cases the single resonance peak of the tuned cascade is replaced by twin resonance peaks. It is seen from the figures that the effect of mistuning on forced response depends upon the engine order of the forcing function and upon the Mach number. For example, in subsonic flow the mistuning has a beneficial effect on torsional response (Fig. 13(a)) for $R=11$, but has an adverse effect (Fig. 13(b)) for $R=39$. This behavior is similar to that noticed in the incompressible flow in Ref. 10. But, this behavior is in contrast to the common belief that mistuning always has an adverse effect on forced response. Therefore, this observation provides an added incentive for pursuing the use of mistuning as a passive control. The decrease in the maximum amplitude of any blade with mistuning for $R=11$ is approximately 51 percent (Fig. 13(a)) and the increase in the maximum amplitude for any blade with mistuning for $R=39$ is approximately 60 percent (Fig. $13(\mathrm{~b})$ ). In supersonic flow for $M=1.1$, the alternating mistuning has an unfavorable effect for both the values of $R=11$ and 39. One of the reasons for this behavior is that the tuned modes $R=11$ and $R=39$ do not have relatively low and high aerodynamic dampings as in the subsonic flow.

\section{CONCLUSIONS}

The analyses of the effects of mistuning on the coupled bending-torsion flutter and response for incompressible flow which were developed in Ref. 10 have been extended into the subsonic and supersonic flows. The following conclusions are reached based on the limited parametric studies presented in this paper.

1. All three regimes of flutter (subcritical, acoustic resonance, and supercritical) were found to exist in both subsonic and supersonic flow for certain parametric combinations. However, supercritical flutter was found only when the Mach number is near unity for the rotor considered herein.

2. It was found that mistuning can have a significant effect on flutter speed depending on the cascade parameters. For Rotor 12 the potential was found to be greater in subsonic than in supersonic flow. For a supersonic fan the effect appears to be sufficient to utilize mistuning as a passive flutter control.

3. The use of alternating structural damping was not found to have any additional benefit over adding damping to all blades equally.
4. The addition of a small amount of structural damping was found to have a much stronger effect for low subsonic Mach numbers than for high subsonic or for all supersonic Mach numbers.

5. As reported by the authors for incompressible flow, the use of uncoupled torsional flutter analys is to deduce the effect of elastic axis position was found to be unreliable. Coupling between bending and torsion, structural damping, and mistuning can change the results significantly in both subsonic and supersonic flows.

6. In general an increase in Mach number was found to have a favorable effect on subcritical flutter in subsonic flow and have an unfavorable effect on supercritical flutter in supersonic flow. Its effect also depends on the bending-to-torsion frequency ratio when the elastic axis is off mid-chord.

7. Mistuning may have either a beneficial or an adverse effect on forced response, depending upon the engine order of the excitation and upon the Mach number.

\section{REFERENCES}

1 Whitehead, D. S., "Torsional Flutter of Unstalled Cascade Blades at Zero Deflection," ARC-R\&M-3429, 1964, Aeronautical Research Council, London, England.

2 Whitehead, D. S., "Effect of Mistuning on the Vibration of Turbomachine Blades Induced by Wakes," Journal of Mechanical Engineering Science, Vol. 8, No. 1, Mar. 1966, pp. 15-21.

3 Hanamura, Y. and Tanaka, H., "A Modification of Flutter Characteristics by Changing Elastic Nature of Neighbouring Blades in Cascades," Proceedings of the 1977 Tokyo Joint Gas Turbine Congress, N. Kikaigakki, ed., Gas Turbine Society of Japan, Tokyo, 1977, pp. 418-427.

4 Ewins, D. J., "An Experimental Investigation of the Forced Vibration of Bladed Dicks Due to Aerodynamic Excitation," Structural Dynamic Aspects of Bladed Disk Assembiies, Proceedings of the Winter Annual Meeting, A. V. Srinivasan, ed., American Society of Mechanical Engineers, New York, 1976, pp. 15-27.

5 Srinivasan, A. V. and Frye, H. M., "Effects of Mistuning on Resonant Stresses of Turbine Blades," Structural Dynamic Aspects of Bladed Disk Assemblies, Proceedings of the Winter Annual Meeting, A. V. Srinivasan, ed., American Society of Mechanical Engineers, New York, 1976, pp. 57-71.

6 Srinivasan, A. V., "Influence of Mistuning on Blade Torsional Flutter, R80-914545-16, United Technologies Research Center, East Hartford, CT, Aug. 1980. (NASA CR-165137).

7 Fabunmi, J. A., "Forced Vibrations of a Single Stage Axial Compressor Rotor," Ph.D. Dissertation, Department of Aeronautics and Astronautics, MIT, Feb. 1978.

8 Cardinale, V. M., Bankhead, H. R., and McKay, R. A., "Experimental Verification of Turboblading Aeromechanics," AGARD 56th Symposium on Turbine Engine Testing, AGARD-CP-293, Advisory Group for Aerospace Research and Development, North Atlantic Treaty Organization, Paris, 1981, pp. 23-1 thru 23-38. 9 Nabatova, N. A. and Shipov, R. A., "Influence of the Material of the Rotor Blades of an AxialFlow Compressor under Flutter Initiating Conditions," Strength of Materials, Vol. 6, No. 8, May 1975, pp. 975-979. Translated from Problemy Prochnosti, Vo 1. 6, August 1974, pp. 63-67. 
10 Kaza, K. R. V. and Kielb, R. E., "Effects of Mistuning on Bending-Torsion Flutter and Response of a Cascade in Incompressible Flow," presented at the AIAA/ASME/ASCE/AHS 22nd Structures, Structural Dynamics and Materials Conference, April 9-10, 1981, Atlanta, Georgia. (also DOE/NASA/1028-29, NASA TM-81674).

11 Smith, S. N., "Discrete Frequency Sound Generation in Axial Flow Turbomachines," ARC-R\&M-3709, 1973, Aeronautical Research Council, London, England. 12 Adamczyk, J. J. and Goldstein, M. E.,

"Unsteady Flow in a Supersonic Cascade with Subsonic Leading-Edge Locus," AIAA Journal, Vol. 16, No. 12, Dec. 1978, pp. 1248-1254.

13 Lane, F., "System Mode Shapes, in the Flutter of Compressor Blade Rows," Journal of the Aeronautical Sciences, Vol. 23, No. 1, Jan. 1956, pp. 54-66. 14 Adamczyk, J. J., NASA-Lewis Research Center, "Private Communication in Calculating the Aerodynamic Coefficients in Supersonic Flow."

15 Whitehead, D. S., "The Effect of Compressibility on Unstalled Torsion Flutter," ARC-R\&M-3754, 1974, Aeronautical Research Council, London, England. 16 Tyler, J. M. and Sofrin, T. G., "Axial Flow Compressor Noise Studies," Society of Automotive Engineers, Trans., Vol. 20, 1962, pp. 309-332.

17 Verdon, J. M., "Further Developments in the Aerodynamic Analysis of Unsteady Supersonic Cascades - Part 2: Aerodynamic Response Predictions," Journal of Engineering for Power, Vol. 99, No. 4, 0ct. 1977, pp. 517-525.

18 Nagashima, T. and Whitehead, D. S., "Linearized Supersonic Unsteady Flow in Cascades," ARC-R\&M-3811, 1977, Aeronautical Research Council, London, England.

19 Bendiksen, 0. and Friedmann, P., "Coupled Bending-Torsion Flutter in a Supersonic Cascade," AIAA Paper No. 80-0701, May 1980.

TABLE I. - PARAMETERS OF NASA TEST ROTOR 12

N

$\mathrm{s} / \mathrm{c}$

$\mu \mathrm{S}$

a

$x_{\alpha_{S}}$

$r_{\alpha_{S}}$

$\xi$

$\omega_{h_{S}} / \omega_{\alpha_{S}}$ (tuned)
56

0.534

258.5

0 (varied in some cases)

0 (varied in some cases)

$0.5774(a=0)$

$0.7638(a=-0.5$ and 0.5$)$

$54.4^{\circ}$

0.357 (varied in some cases) 


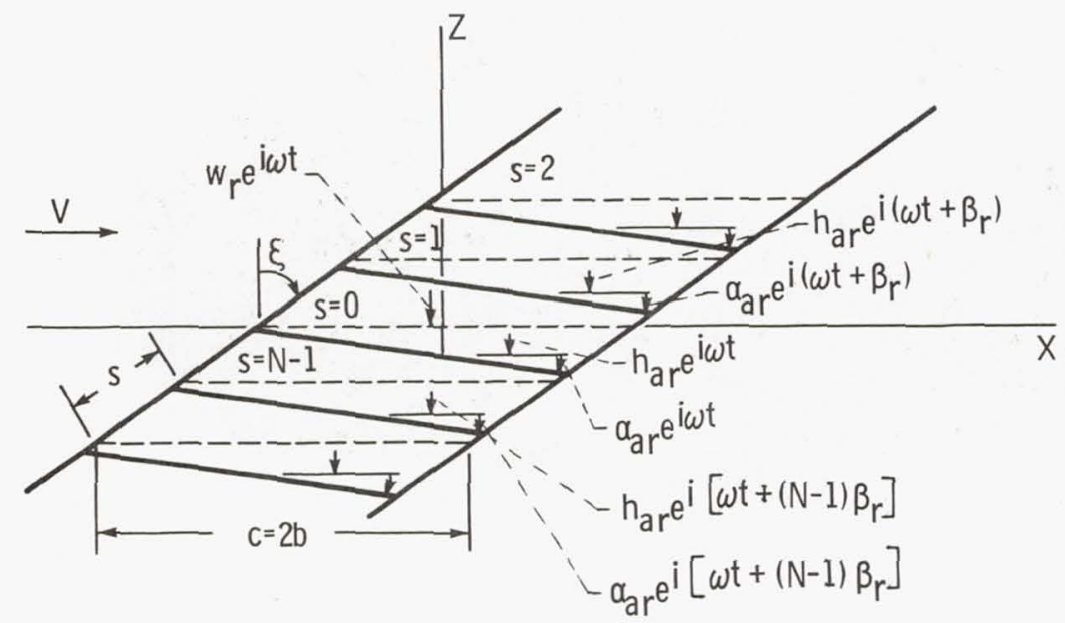

Figure 1. - Geometry of a tuned cascade in rth mode (note that the variable $s$ represents the gap between the chords as well as the blade number index).

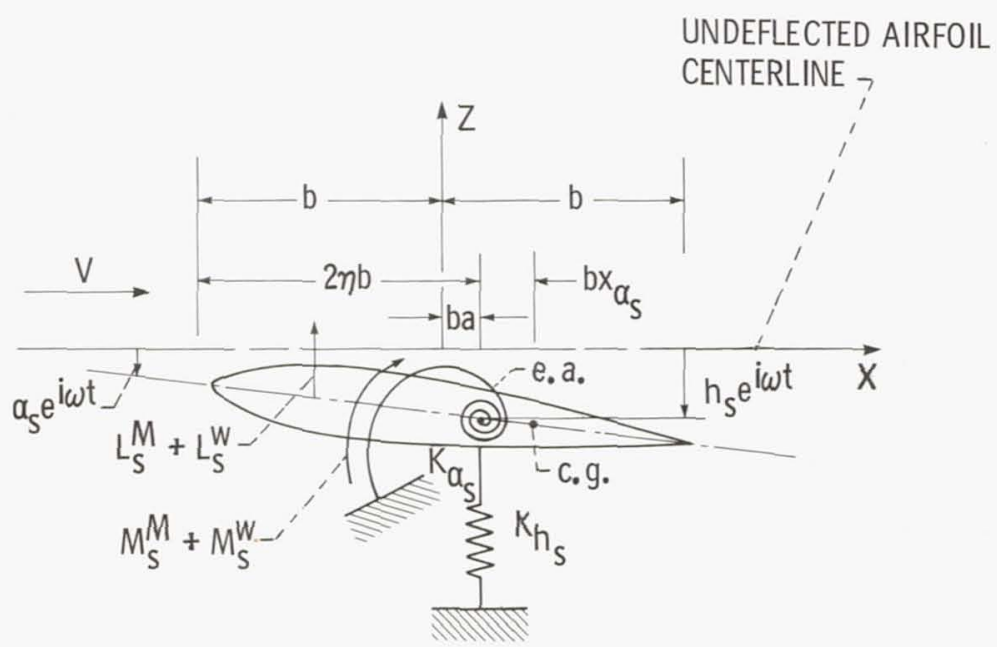

Figure 2. - Airfoil restrained from bending and torsional motion (sinusoidal wakes not shown). 


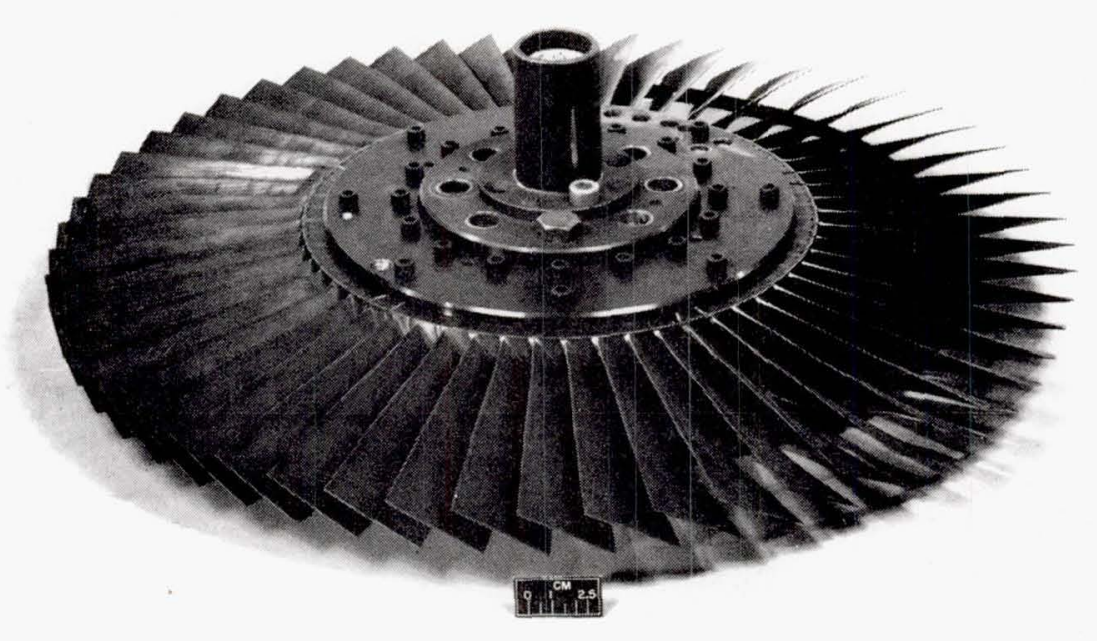

C-71-1356

Figure 3. - NASA Test Rotor 12.

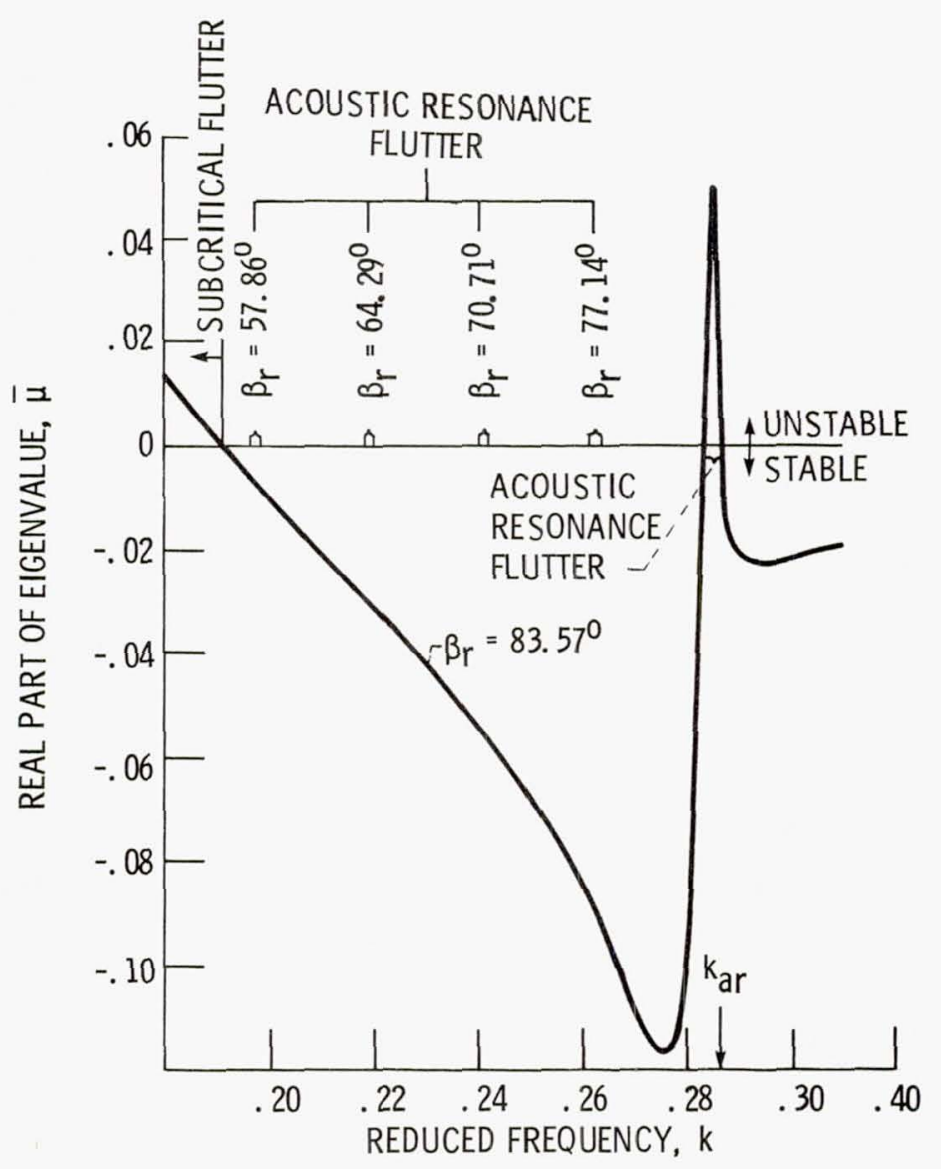

Figure 4. - Subcritical and acoustic resonance flutter predictions of a tuned cascade: $M=0.85, a=0$, $x_{\alpha_{s}}=0, \omega_{h_{s}} / \omega_{\alpha_{s}}=0.357$. 


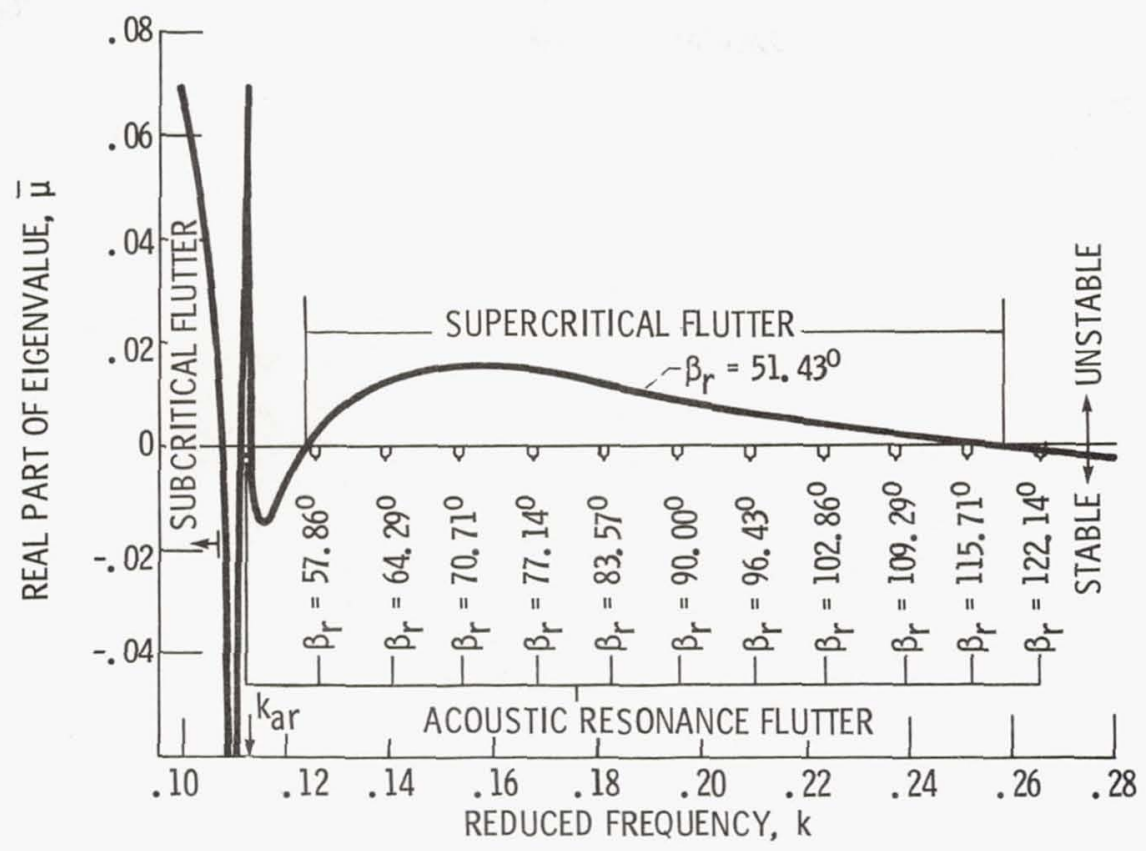

Figure 5. - Subcritical, supercritical, and acoustic resonance flutter predictions of a tuned cascade: $M=0.9, a=0, x_{\alpha_{s}}=0, \omega_{h_{s}} l \omega_{\alpha_{s}}=$

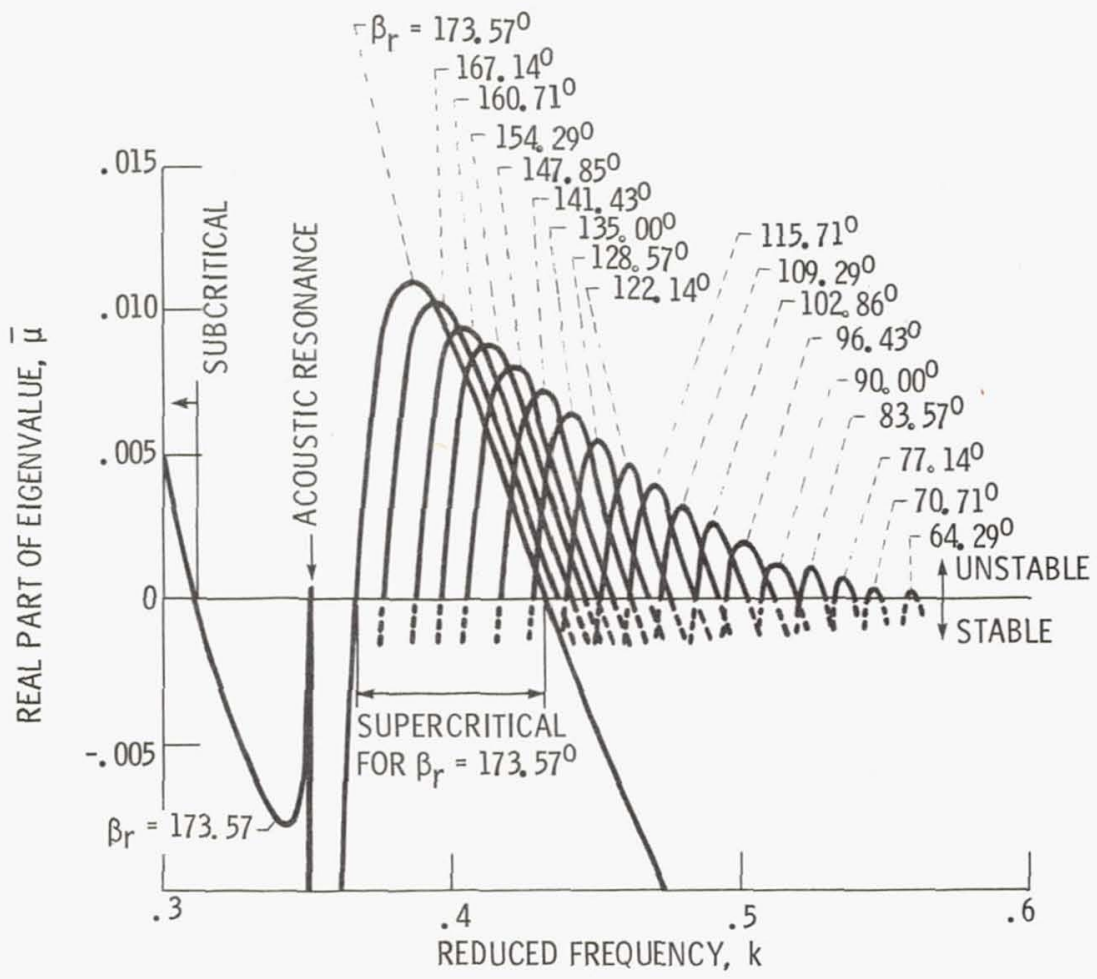

Figure 6. - Subcritical, acoustic resonance, and supercritical flutter predictions of a tuned cascade: $M=1.1, a=0, x_{\alpha_{s}}=0$, $\omega_{h_{s}} / \omega_{\alpha_{s}}=0.357$, undamped. 

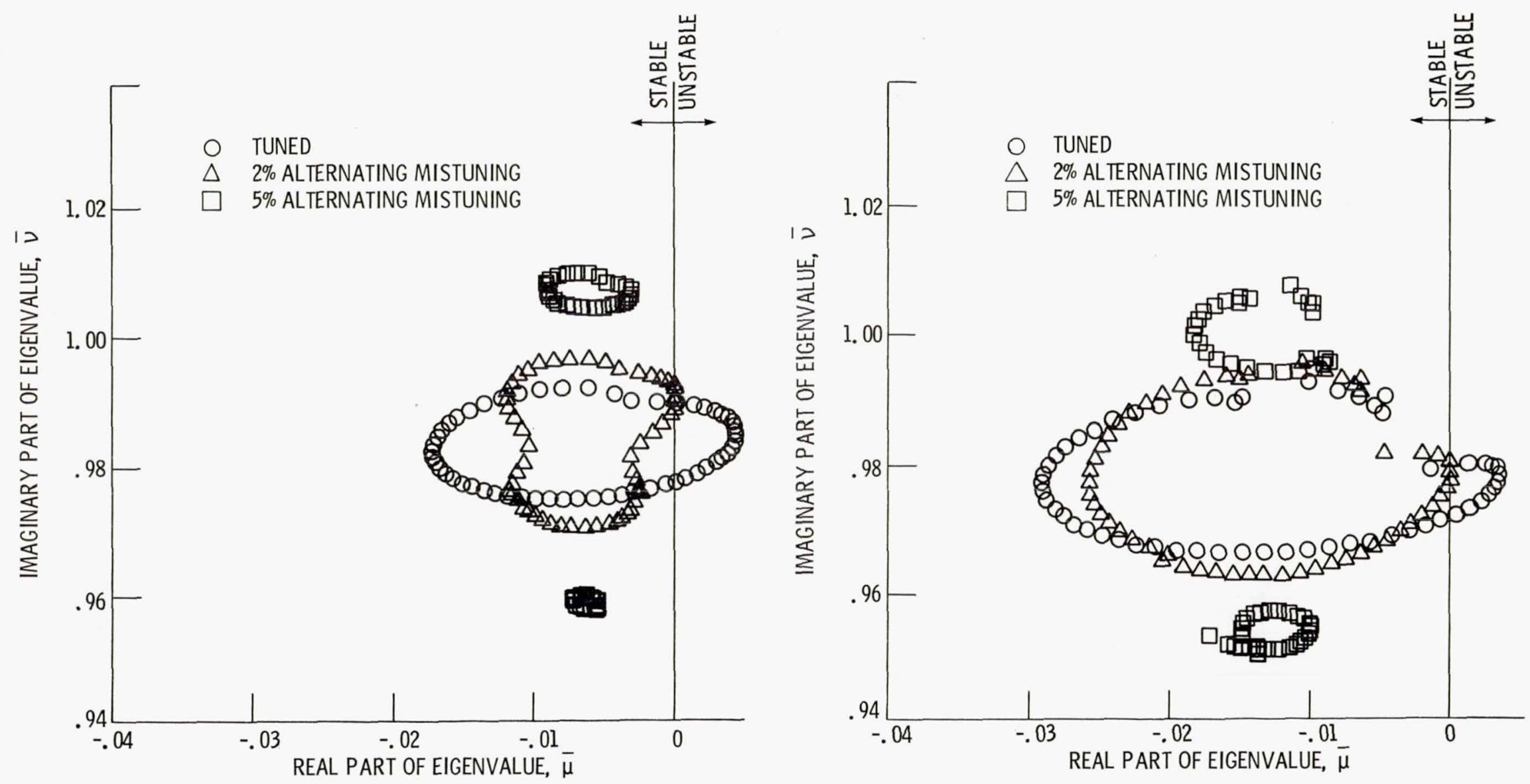

Figure 7. - Effect of alternating mistuning on eigenvalues: $M=0, x_{\alpha_{s}}=0, k=0.5086, \omega_{h_{s}} / \omega_{0}=0.357$, undamped.

Figure 8. - Effect of alternating mistuning on eigenvalues in subsonic flow: $M=0.5, a=0, x_{a_{s}}=0, k=0.4694, \omega_{h_{s}} l \omega_{0}=0.357$, undamped. 

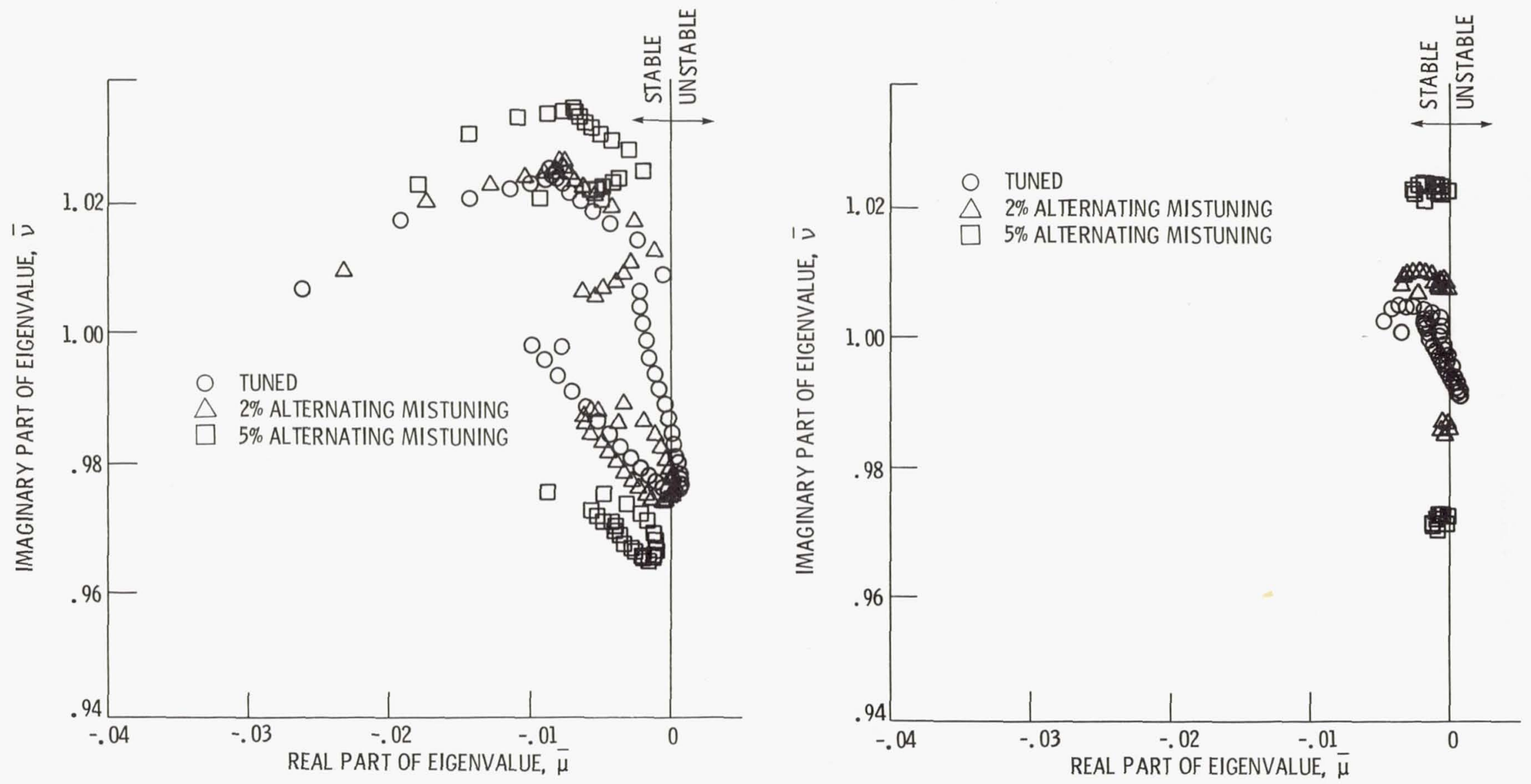

Figure 9. - Effect of alternating mistuning on eigenvalues in supersonic flow: $M=1.15, a=0, x_{\alpha_{s}}=0, k=0.4361, \omega_{h_{s}} / \omega_{0}=0.357$, undamped.

Figure 10. - Effect of alternating mistuning on eigenvalues in supersonic flow: $M=1.4, a=0, x_{\alpha_{s}}=0, k=0.6606, \omega_{h_{s}} / \omega_{0}=0.357$, un-
damped. 


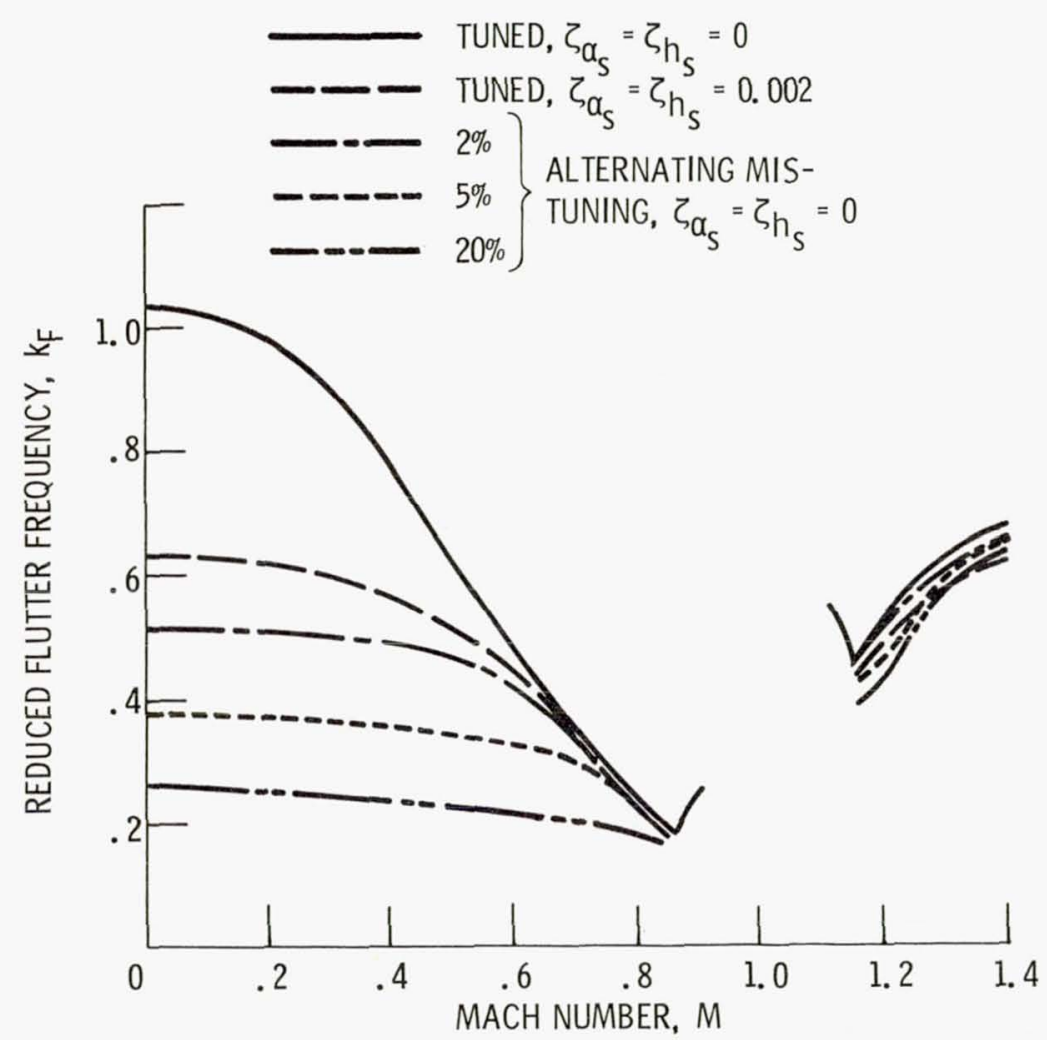

Figure 11. - Effect of compressibility, mistuning and structural damping on the reduced flutter frequency: $a=0, x_{\alpha_{s}}=0$, $\omega_{\mathrm{h}_{\mathrm{s}}} / \omega_{0}=0.357$.

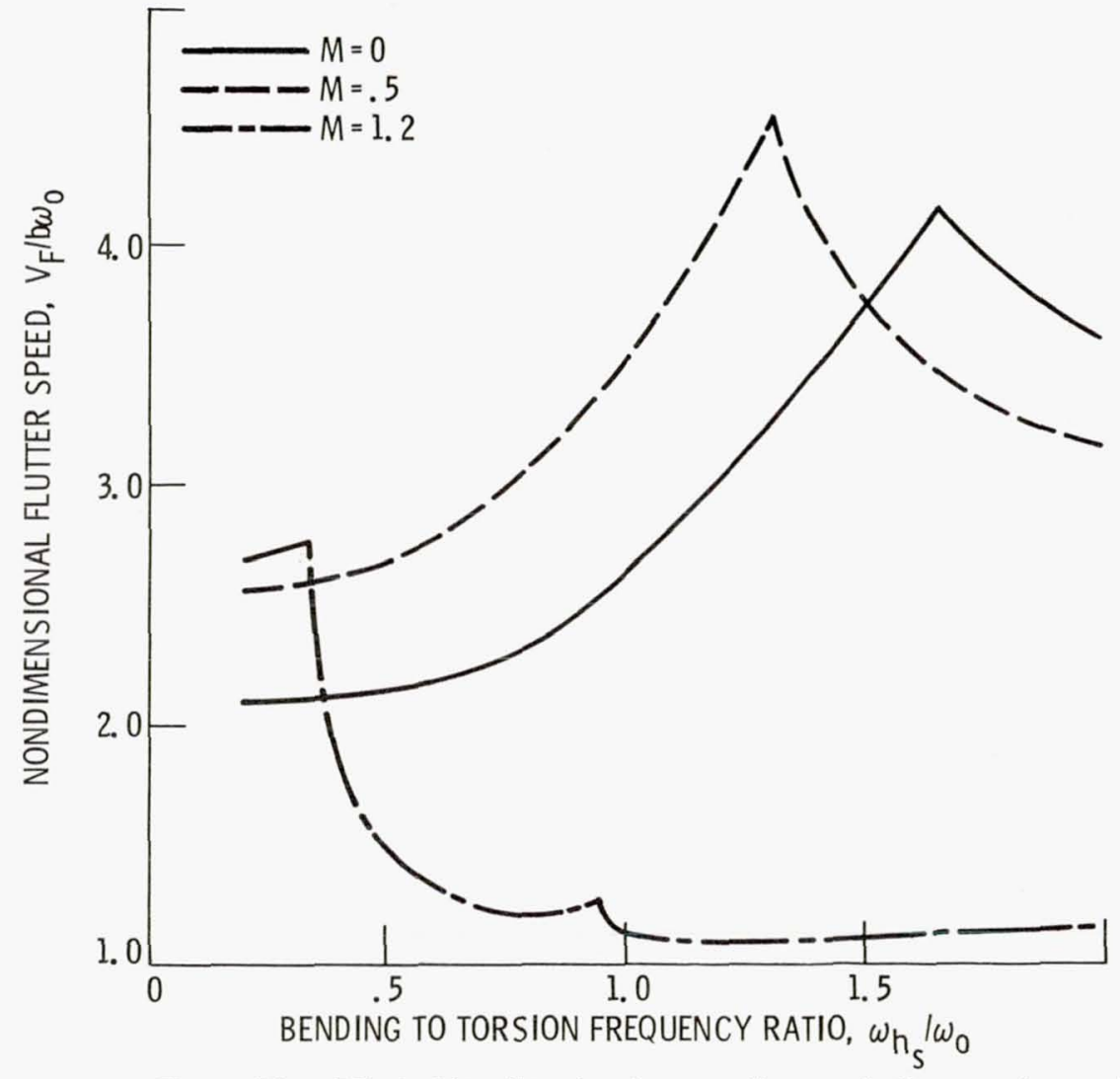

Figure 12. - Effect of bending-torsion coupling on flutter speed: $a=.5$, $x_{a_{s}}=0.5, \zeta_{h_{s}}=\zeta_{a_{s}}=0.002$, tuned. 


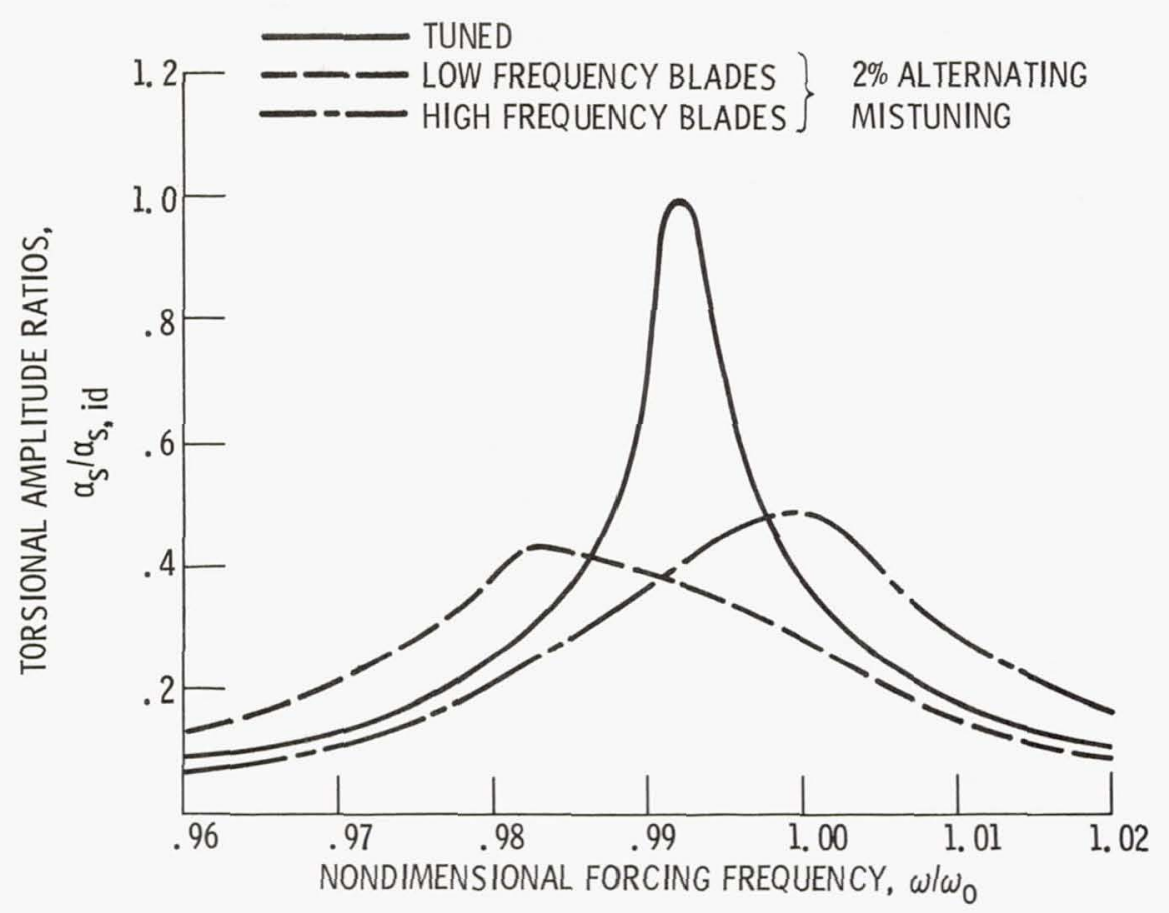

Figure 13(a). - Effect of blade alternating mistuning on coupled bending torsion response: $M=0.5, R=11, \zeta_{h_{s}}=\zeta_{a_{s}}=0.002$, $\omega_{\mathrm{h}_{\mathrm{s}}} / \omega_{0}=0.357$.

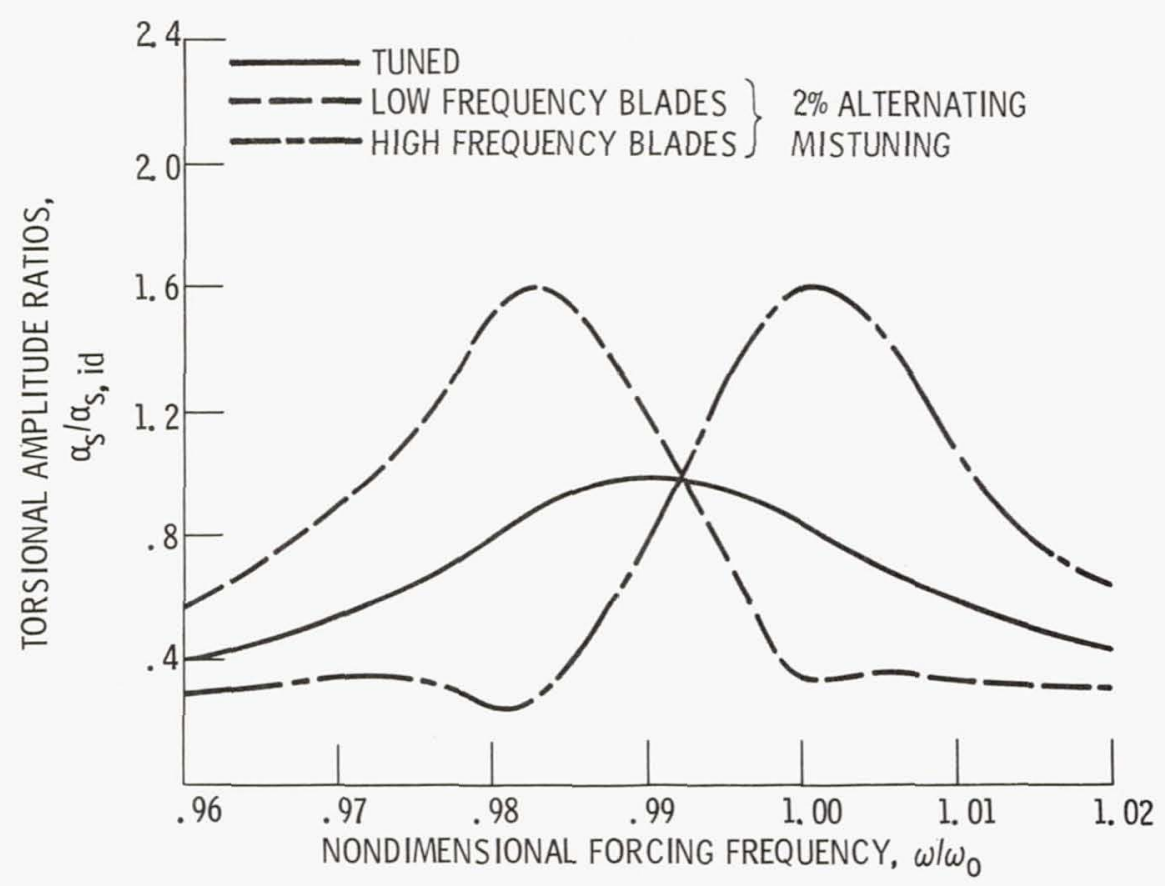

Figure 13(b). - Effect of blade alternating mistuning on coupled bending torsion response: $M=0.5, R=39, \zeta_{h_{s}}=\zeta_{a_{s}}=0.002$, $\omega_{h_{\mathrm{s}}} l \omega_{0}=0.357$. 


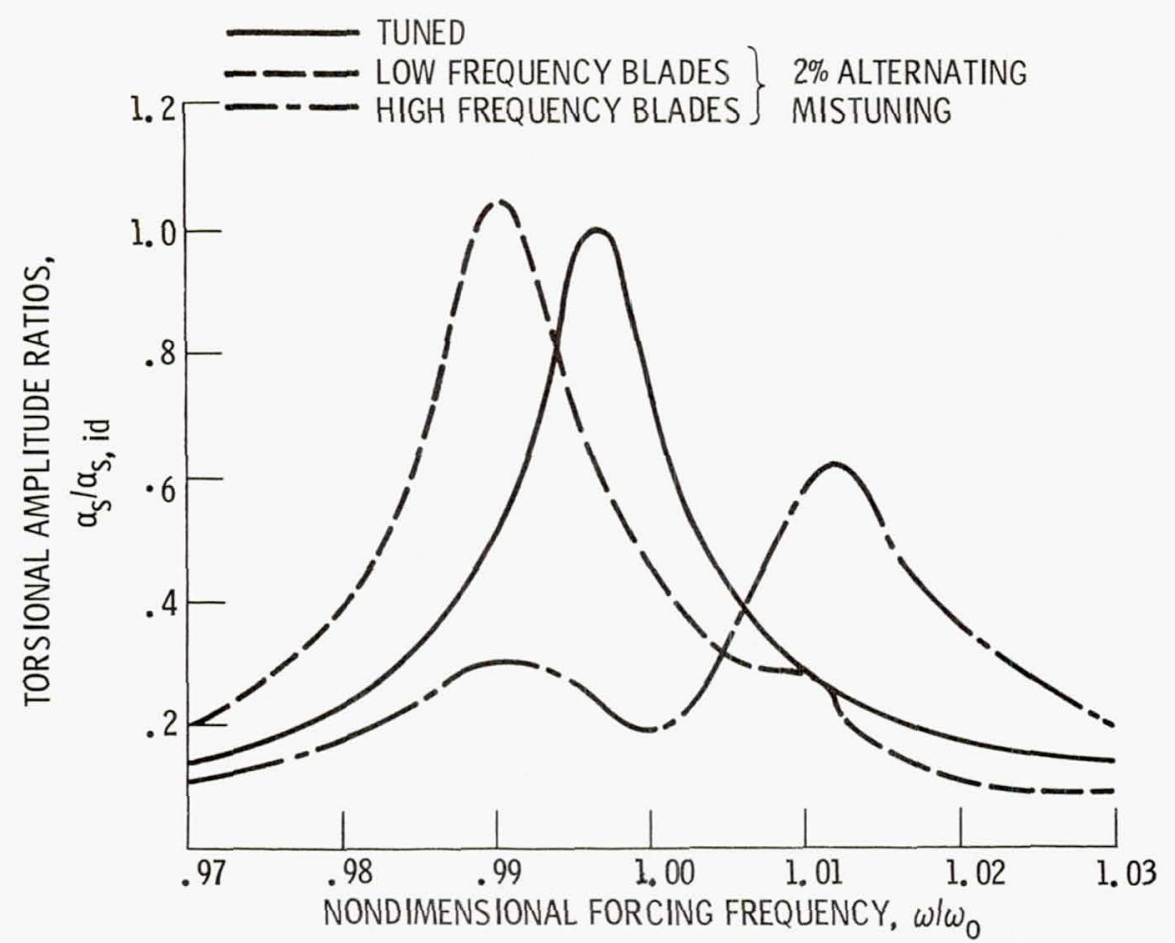

Figure 14(a). - Effect of blade alternating mistuning on coupled bending torsion response: $M=1.1, R=11, \zeta_{h_{s}}=\zeta_{a_{s}}=0.002$, $\omega_{\mathrm{h}_{\mathrm{s}}} / \omega_{0}=0.357$.
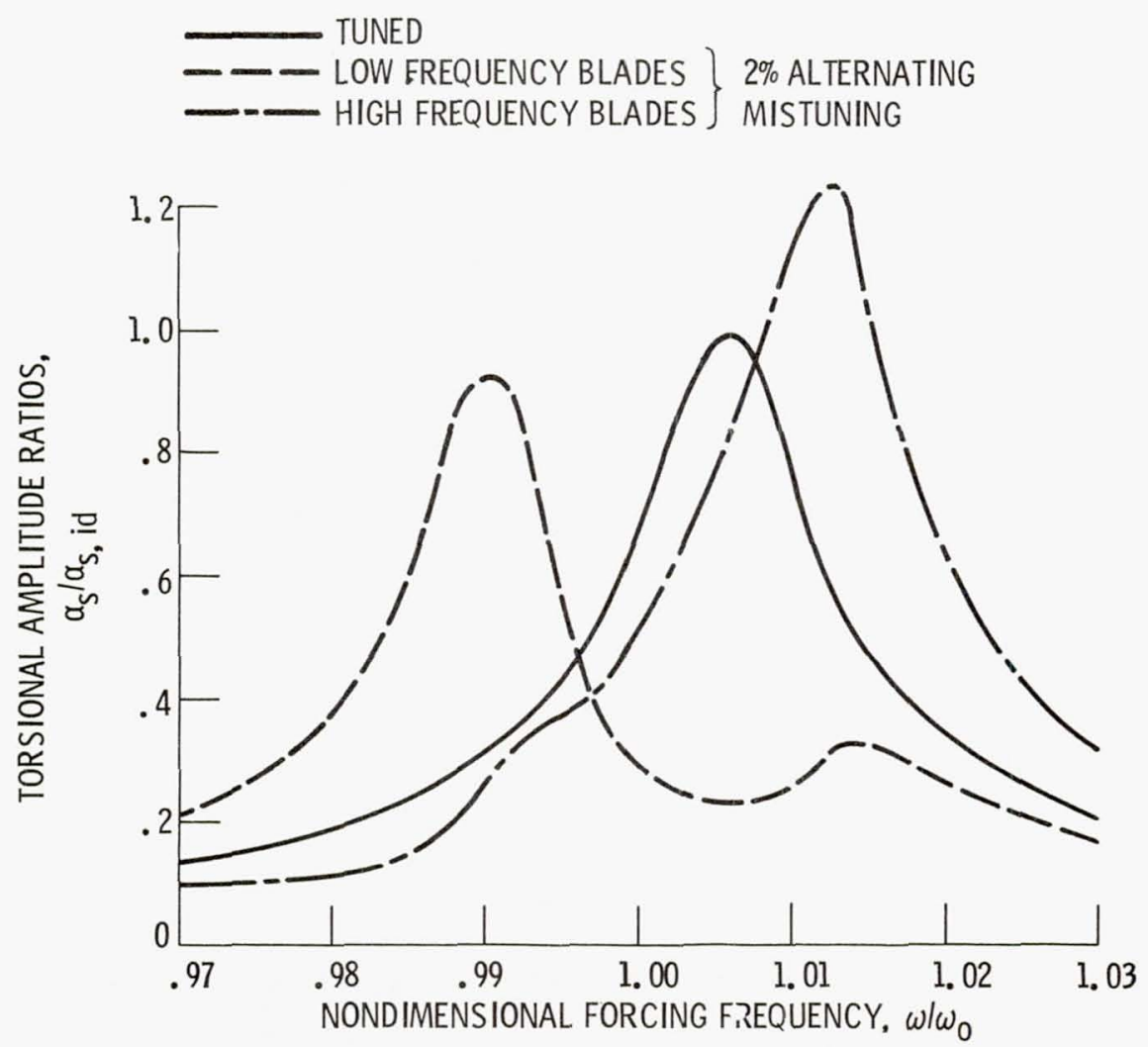

Figure 14(b). - Effect of blade alternating mistuning on coupled bending torsion response: $M=1.1, \mathrm{R}=39, \zeta_{\mathrm{h}_{\mathrm{s}}}=\zeta_{\alpha_{\mathrm{s}}}=0.002$, $\omega_{\mathrm{h}_{\mathrm{s}}} / \omega_{\mathrm{O}}=0.357$. 


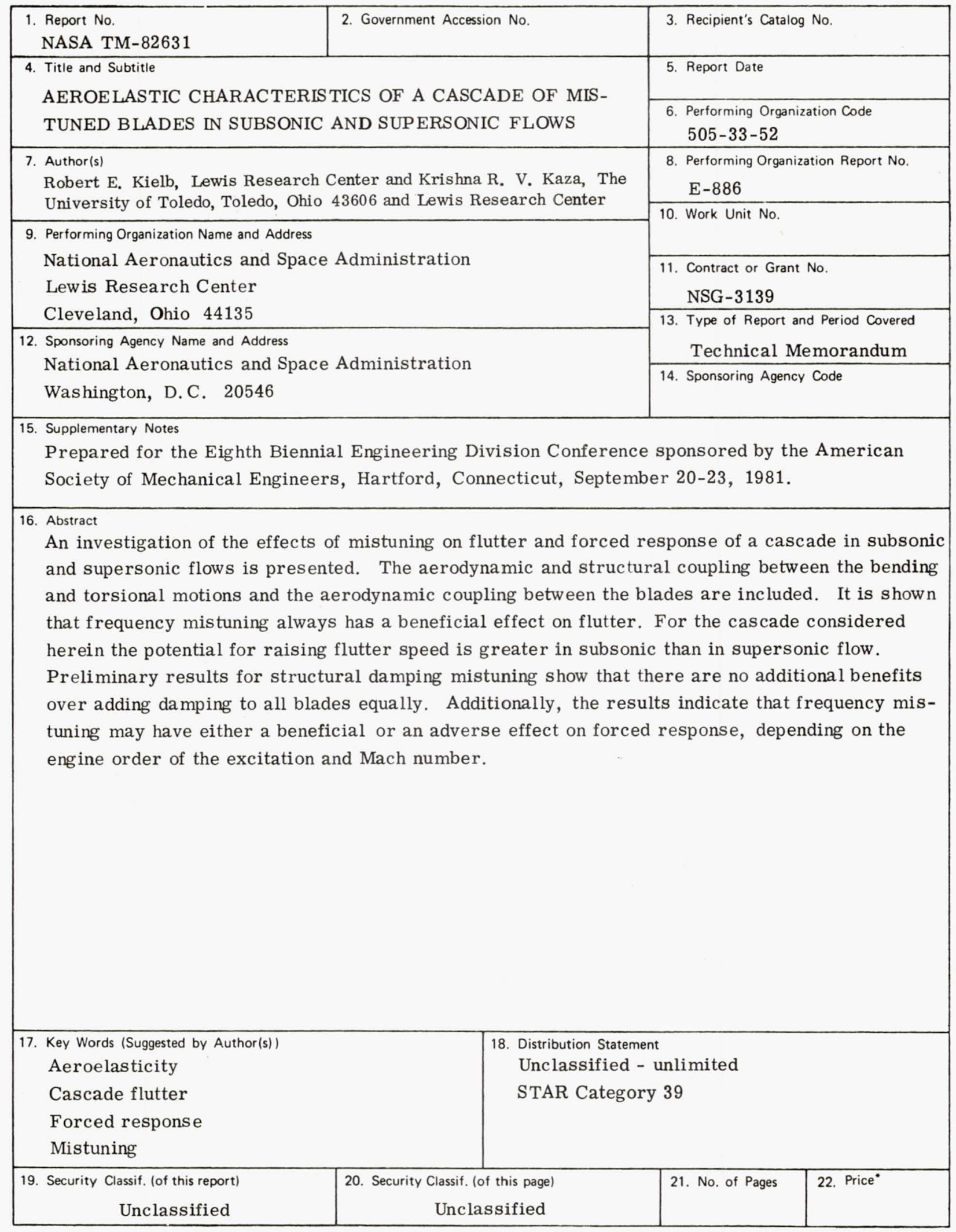

${ }^{*}$ For sale by the National Technical Information Service, Springfield, Virginia 22161 
NATIONAL AERONAUTICS AND SPACE ADMINISTRATION WASHINGTON. D.C. 20546

OFFICIAL BUSINESS

PENALTY FOR PRIVATE USE:300 SPECIAL FOURTH-CLASS RATE BOOK

POSTAGE AND FEES PAID NATIONAL AERONAUTICS AND SPACE ADMINISTRATION

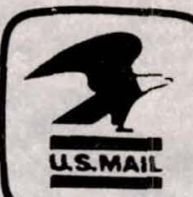

